Pacific Journal of Mathematic 


\title{
NILPOTENT APPROXIMATIONS AND QUASINILPOTENT OPERATORS
}

\section{Constantin Apostol and Norberto Salinas}

\begin{abstract}
Let $\mathscr{H}$ be a separable, infinite dimensional, complex Hilbert space and let $\mathscr{L}(\mathscr{H})$ denote the algebra of all (bounded, linear) operators on $\mathscr{H}$. This paper is concerned with some aspects of the uniform approximation of an operator in $\mathscr{L}(\mathscr{H})$ by nilpotent operators.
\end{abstract}

If $T$ is an operator in $\mathscr{L}(\mathscr{H})$ we shall denote by $\sigma(T)$ the spectrum of $T$ and $r(T)$ the spectral radius of $T$. We recall that an operator $T$ in $\mathscr{L}(\mathscr{H})$ is said to be quasinilpotent if $\sigma(T)=\{0\}$ or equivalently $r(T)=\lim _{n \rightarrow \infty}\left\|T^{n}\right\|^{1 / n}=0$. In [5] [7] it wasproved that a quasinilpotent operator $T$ in $\mathscr{L}(\mathscr{H})$ is the uniform limit of a sequence $\left\{Q_{k}\right\}$ of nilpotent operators on $H$ (cf. [6, Problem 7]). For each positive integer $k$ let $o_{k}$ be the order of the nilpotent operator $Q_{k}$, that is $o_{k}$ is the smallest positive integer such that $Q_{k}^{o k}=0$. In view of the result mentioned above it is natural to ask whether there exists any relationship between the rate of decrease of the sequence $\left\|T^{o_{k}}\right\|^{1 / o_{k}}$ and the rate of decrease of the sequence $\left\|T-Q_{k}\right\|$. Furthermore, it is reasonable to expect that there exists a characterization of the set of quasinilpotent operators in terms of its nilpotent approximations. These questions seem to be rather hard and in this paper we present some insights into these problems (cf. Corollary 3.4).

In Theorem 3.5 we prove that the distance from an arbitrary operator $T$ in $\mathscr{L}(\mathscr{H})$ to the set $\mathscr{N}(\mathscr{H})$ of all nilpotent operators in $\mathscr{L}(\mathscr{H})$ is at most $r(T)$ and in Theorem 3.1 we give precise estimates for the nilpotent approximations of $T$ in the case that $T$ is a biquasitriangular operator and zero is in the essential spectrum of $T$ (see $\S 3$ for the corresponding definitions). Another by-product of our discussion (cf. Proposition 4.4) is that if $T$ is an operator on $\mathscr{H}$ such that $\liminf _{n \rightarrow \infty} \sqrt{n}\left\|T^{n}\right\|^{1 / n}=0$, then the quasinilpotent operator $T$ is actually pseudonilpotent (cf. [10, Problem 1]).

We are indebted to D. A. Herrero for his very useful comments, specifically, for his suggestions about the proof of Theorem 3.5 and for providing us with the example of Remark 4.5.

2. The central lemma. The following lemma is central to our purposes.

Lemma 2.1. Let $T \in \mathscr{L}(\mathscr{H})$. Then for every $\alpha>0, \beta>r(T)$ 
and every positive integer $n$ there exists $Q \in \mathscr{N}(\mathscr{H} \oplus \mathscr{H})$ of order at most $n$ (i.e. $Q^{n}=0$ ) such that

$$
\|(T \oplus 0)-Q\|<\alpha\|T\|+\beta+\frac{\left\|T^{n}\right\|}{\alpha \beta^{n-1}} .
$$

Proof. Let $U$ and $V$ be two isometries in $\mathscr{L}(\mathscr{H})$ such that $V V^{*}=1-U U^{*}$. We define for $1 \leqq k \leqq n$ the subspace $\mathscr{C}_{k}$ of $\mathscr{H} \oplus \mathscr{H}$ given by $\mathscr{N}_{k}=\left\{T^{k-1} x \oplus \alpha \beta^{k-1} V^{k-1} U x, x \in \mathscr{H}\right\}$. Note that $\mathscr{N}_{k}$ is closed because it is the image under the isometry $\left(\begin{array}{ll}1 & 0 \\ 0 & V^{k-1} U\end{array}\right)$ of the graph of the transpose of $T^{k-1} /\left(\alpha \beta^{k-1}\right)$. It is easy to see that $\mathscr{A}_{j} \cap \mathscr{M}_{k}=\{0\}$ if $1 \leqq j, k \leqq n, j \neq k$. This is due to the fact that the second components of the elements in $\mathscr{K}_{j}$ and $\mathscr{N}_{k}$ are orthogonal. Let $\mathscr{M}=\sum_{k=1}^{n} \mathscr{\mathscr { K }}_{k}=\left\{y_{1}+\cdots+y_{n}, y_{k} \in \mathscr{M}_{k}, 1 \leqq k \leqq n\right\}$. Now we prove that $\mathscr{M}$ is closed. Let $\left\{y_{m}\right\}$ be a sequence in $\mathscr{M}$ s.t. $\lim _{m \rightarrow \infty} y_{m}=0$. Since $\mathscr{K}_{j} \cap \mathscr{N}_{k}=\{0\}, j \neq k$, we can write uniquely $y_{m}=\sum_{k=1}^{n} y_{m, k}$, where $y_{m, k}=T^{k-1} x_{m, k} \oplus \alpha \beta^{k-1} V^{k-1} U x_{m, k}$, for some $x_{m, k} \in \mathscr{H}, 1 \leqq k \leqq n$, $m=1,2, \cdots$. Since $\lim _{m \rightarrow \infty} y_{m}=0$, then $\lim _{m \rightarrow \infty} \sum_{k=1}^{n} \alpha \beta^{k-1} V^{k-1} U x_{m, k}=0$. Then $\lim _{m \rightarrow \infty} V^{k-1} U x_{m, k}=0,1 \leqq k \leqq n$ and hence $\lim _{m \rightarrow \infty} x_{m, k}=0$, $1 \leqq k \leqq n$. Therefore, $\lim _{m \rightarrow \infty} y_{m, k}=0,1 \leqq k \leqq n$ and hence $\mathscr{C}$ is closed. Now we define $Q \in \mathscr{N}(\mathscr{H} \oplus \mathscr{H})$ by

$$
Q\left|\mathscr{M}_{n} \oplus \mathscr{C}^{\perp}=0, Q\right| \sum_{k=1}^{n-1} \mathscr{M}_{k}=(T \oplus \beta V) \mid \sum_{k=1}^{n-1} \mathscr{M}_{k} .
$$

Thus, the representing matrix of $Q \mid \mathscr{C}$ on $\sum_{k=1}^{n} \mathscr{M}_{k}$ is of the form

$$
\left(\begin{array}{cccccc}
0 & 0 & 0 & \cdots & 0 & 0 \\
* & 0 & 0 & \cdots & 0 & 0 \\
0 & * & 0 & \cdots & 0 & 0 \\
\vdots & & & & \\
0 & 0 & 0 & \cdots & * & 0
\end{array}\right) .
$$

Therefore it is clear that $Q^{n}=0$. Let $P_{n}$ be the projection onto kernel $V^{* n}$. Then

$$
\begin{aligned}
& {\left[\left(T \oplus \beta P_{n} V\right)-Q\right] \sum_{k=1}^{n}\left(T^{k-1} x_{k} \oplus \alpha \beta^{k-1} V^{k-1} U x_{k}\right) } \\
&= {\left[T \sum_{k=1}^{n-1} T^{k-1} x_{k}-T \sum_{k=1}^{n-1} T^{k-1} x_{k}\right] \oplus \beta P_{n} V \sum_{k=1}^{n} \alpha \beta^{k-1} V^{k-1} U x_{k} } \\
&-\beta V \sum_{k=1}^{n} \alpha \beta^{k-1} V^{k-1} U x_{k} \\
&= T^{n} x_{n} \oplus\left[P_{n} \alpha \beta^{n} V^{n} U x+\sum_{k=1}^{n-1} \alpha \beta^{k}\left(P_{n}-1\right) V^{k} U x_{k}\right] \\
&= T^{n} x_{n} \oplus 0 .
\end{aligned}
$$


Since

$$
\begin{aligned}
\left\|x_{n}\right\| & =\frac{1}{\alpha \beta^{n-1}}\left\|\alpha \beta^{n-1} V^{n-1} U x_{n}\right\| \leqq \frac{1}{\alpha \beta^{n-1}}\left\|\sum_{k=1}^{n} \alpha \beta^{k-1} V^{k-1} U x_{k}\right\| \\
& \leqq \frac{1}{\alpha \beta^{n-1}}\left\|\sum_{k=1}^{n} T^{k} x_{k} \oplus \alpha \beta^{k-1} V^{k-1} U x_{k}\right\| .
\end{aligned}
$$

We conclude that $\left\|\left[\left(T \oplus \beta P_{n} V\right)-Q\right] \mid \mathscr{C}\right\| \leqq\left\|T^{n}\right\| / \alpha \beta^{n-1}$. From now on given a subspace $\mathscr{X}$ of $\mathscr{H}, P_{\mathscr{Z}}$ will denote the projection from $\mathscr{H}$ onto $\mathscr{X}$. Then

$$
\begin{aligned}
\|(T \oplus 0)-Q\| \leqq & \left\|(T \oplus 0) P_{\mathscr{M}} \perp\right\|+\left\|[(T \oplus 0)-Q] P_{\mathscr{A}}\right\| \\
\leqq & \|T\|\left\|P_{\mathscr{C} \oplus 0} P_{\mathscr{M}^{\perp}}\right\|+\left\|\left[\left(T \oplus \beta P_{n} V\right)-Q\right] P_{\mathscr{M}}\right\| \\
& +\left\|0 \oplus \beta P_{n} V\right\| \leqq\|T\|\left\|P_{\mathscr{C} \oplus 0} P_{\mathscr{M}_{1}^{\perp}}\right\| \\
& +\beta+\left\|T^{n}\right\| / \alpha \beta^{n-1} .
\end{aligned}
$$

Thus in order to complete the proof it suffices to show that $\left\|P_{\mathscr{K} \oplus 0} P_{\mathscr{M}_{1}^{\perp}}\right\| \leqq \alpha$. Notice that $\mathscr{M}_{1}^{\perp}=\{y \oplus z:\langle y, x\rangle+\alpha\langle z, U x\rangle=0$ for all $x \in \mathscr{H}\}$. Hence $\mathscr{A}_{1}^{\perp}=\left\{\left(-\alpha U^{*} z\right) \oplus z, z \in \mathscr{H}\right\}$. Therefore $\left\|P_{\mathscr{C} \oplus 0} P_{\mathscr{M}_{1}^{\perp}}\right\|=\left\|\left.P_{\mathscr{C} \oplus 0}\right|_{\mathscr{M}_{1}^{\perp}}\right\| \leqq \alpha$.

Following [9] we shall denote by $R_{e}(T)$ the reducing essential spectrum of the operator $T$, that is $R_{e}(T)$ is the set of complex numbers $\lambda$ such that there exists a projection $P$ in $\mathscr{L}(\mathscr{H})$ of infinite rank and nullity such that $(T-\lambda) P$ and $\left(T^{*}-\bar{\lambda}\right) P$ are compact operators.

THEOREM 2.2. Let $T$ be in $\mathscr{L}(\mathscr{H})$ such that $R_{e}(T) \neq \varnothing$ and suppose that $0 \in R_{e}(T)$. Then for every $\alpha>0, \beta>r(T), \gamma>1$ and every positive integer $n$ there exists $Q \in \mathscr{N}(\mathscr{H})$ of order at most $n$ such that

$$
\|T-Q\| \leqq \gamma\left(\alpha\|T\|+\beta+\frac{\left\|T^{n}\right\|}{\alpha \beta^{n-1}}\right)
$$

Proof. From [9, Theorem 4.6] it follows that for every $\varepsilon>0$ there exists a unitary transformation $U_{\varepsilon}: \mathscr{H} \rightarrow \mathscr{H} \oplus \mathscr{H}$ such that $\left\|U_{\varepsilon} T U_{\varepsilon}^{*}-(T \oplus 0)\right\|<\varepsilon$. On the other hand, from Lemma 2.1 we deduce that there exists a nilpotent operator $Q^{\prime}$ on $\mathscr{H} \oplus \mathscr{H}$ of order at most $n$ such that $\left\|T \oplus 0-Q^{\prime}\right\| \leqq \alpha\|T\|+\beta+\left\|T^{n}\right\| /\left(\alpha \beta^{n-1}\right)$. Letting now $Q=U_{\varepsilon}^{*} Q^{\prime} U_{\varepsilon}$ we observe that $\|T-Q\|=\left\|U_{\varepsilon} T U_{\varepsilon}^{*}-Q^{\prime}\right\| \leqq$ $\varepsilon+\left\|(T \oplus 0)-Q^{\prime}\right\| \leqq \varepsilon+\alpha\|T\|+\beta+\left\|T^{n}\right\| /\left(\alpha \beta^{n-1}\right)$. The proof of the theorem is completed by choosing $\varepsilon$ small enough.

3. Nilpotent approximation of biquasitriangular operators. In what follows, given an operator $T$ in $\mathscr{L}(\mathscr{H})$ we denote by $E(T)$ 
the essential spectrum of $T$, i.e. $E(T)=\{\lambda \in \sigma(T): T-\lambda$ is not Fredholm \}. Also $E_{l}(T)$ and $E_{r}(T)$ will denote the left essential spectrum and the right essential spectrum of the operator $T$, that is $E_{l}(T)=\{\lambda \in E(T): T-\lambda$ is not semi-Fredholm with $\operatorname{dim}[\operatorname{null}(T-\lambda)]<\infty\}$ and $E_{r}(T)=\left[E_{l}\left(T^{*}\right)\right]^{*}$.

Following [6] we say that an operator $T$ on $\mathscr{H}$ is quasitriangular if there exists an increasing sequence $\left\{P_{n}\right\}$ of finite rank projections in $\mathscr{L}(\mathscr{L})$ tending strongly to the identity operator such that $\lim _{n \rightarrow \infty}\left\|\left(1-P_{n}\right) T P_{n}\right\|=0$ and we say that $T$ is biquasitriangular if both $T$ and $T^{*}$ are quasitriangular [2]. In [3] it was shown that $T$ is biquasitriangular if and only if the index of $T-\lambda$ is zero for every complex number $\lambda$ such that $T-\lambda$ is semi-Fredholm. In particular, if $T$ is biquasitrianglar $E(T)=E_{l}(T) \cap E_{r}(T)$.

In the following theorem we give some precise estimates for the nilpotent approximation of a biquasitriangular operator.

THEOREM 3.1. Let $T$ in $\mathscr{L}(\mathscr{H})$ be a biquasitriangular operator and suppose that $0 \in E(T)$. Then for every $\alpha>0, \beta>r(T), \gamma>1$ and every positive integer $n$ there exists a nilpotent operator $Q$ in $\mathscr{L}(\mathscr{H})$ of order at most $4 n$ such that $(*)$ is valid.

Proof. From [11, Proposition 3.2] it follows that for every $\varepsilon>0$ there exists a unitary transformation $V_{\varepsilon}: \mathscr{H} \rightarrow \mathscr{H} \oplus \mathscr{H} \oplus \mathscr{H} \oplus \mathscr{H}$ and a compact operator $K_{\varepsilon}$ in $\mathscr{L}(\mathscr{H})$ with $\left\|K_{\varepsilon}\right\|<\varepsilon$ such that

$$
V_{\varepsilon}\left(T-K_{\varepsilon}\right) V_{\varepsilon}^{*}=\left(\begin{array}{llll}
S_{1} & 0 & 0 & 0 \\
L_{21} & S_{2} & 0 & 0 \\
L_{31} & L_{32} & S_{3} & 0 \\
L_{41} & L_{42} & L_{43} & S_{4}
\end{array}\right)
$$

where $L_{i j} \in \mathscr{L}(\mathscr{H}), 1 \leqq j<i \leqq 4$, and for each $1 \leqq j \leqq 4, S_{j}$ is an operator in $\mathscr{L}(\mathscr{H})$ such that $S_{j}$ is unitarily equivalent to $M_{j} \oplus N_{j}$ where $M_{j}$ is a block diagonal operator on $\mathscr{H}$ with $E\left(M_{j}\right) \subset E(T)$ and $N_{j}$ is a normal operator in $\mathscr{L}(\mathscr{H})$ such that $E\left(N_{j}\right)=E(T)$. Since $E\left(N_{j}\right)=R_{e}\left(N_{j}\right)$ [9, Theorem 3.10], $0 \in E(T)$ and $R_{e}\left(M_{j} \oplus N_{j}\right)=$ $R_{e}\left(M_{j}\right) \cup R_{e}\left(N_{j}\right)$ [9, Lemma 4.10], it follows that $0 \in R_{e}\left(S_{j}\right), 1 \leqq j \leqq 4$. Now we chose $\varepsilon>0$ small enough so that $r\left(T-K_{\varepsilon}\right)<\beta$. Since $\left\|S_{j}^{k}\right\| \leqq\left\|\left(T-K_{\varepsilon}\right)^{k}\right\|, 1 \leqq j \leqq 4, k=1,2, \cdots$, we also have $r\left(S_{j}\right)<\beta$, $1 \leqq j \leqq 4$. Given a fixed number $\delta$ with $1<\delta<\gamma$, from Theorem 2.2 we can find nilpotent operators $Q_{j}$ in $\mathscr{L}(\mathscr{H})$ of order at most $n$ such that $\left\|S_{j}-Q_{j}\right\| \leqq \delta\left(\alpha\left\|S_{j}\right\|+\beta+\left\|S_{j}^{n}\right\| / \alpha \beta^{n-1}\right), \quad 1 \leqq j \leqq 4$. Now let $Q$ be the operator in $\mathscr{L}(\mathscr{H})$ defined by 


$$
V_{\varepsilon} Q V_{\varepsilon}^{*}=\left(\begin{array}{llll}
Q_{1} & 0 & 0 & 0 \\
L_{21} & Q_{2} & 0 & 0 \\
L_{31} & L_{32} & Q_{3} & 0 \\
L_{41} & L_{42} & L_{43} & Q_{4}
\end{array}\right)
$$

It is obvious that $Q$ is a nilpotent operator of order at most $4 n$ and we see that

$$
\begin{aligned}
\left\|T-K_{\varepsilon}-Q\right\| & =\left\|V_{\varepsilon}\left(T-K_{\varepsilon}-Q\right) V_{\varepsilon}^{*}\right\|=\max _{1 \leqq j \leqq 4}\left\|S_{j}-Q_{j}\right\| \\
& \leqq \delta\left(\alpha\left\|T-K_{\varepsilon}\right\|+\beta+\frac{\left\|\left(T-K_{\varepsilon}\right)^{n}\right\|}{\alpha \beta^{n-1}}\right) .
\end{aligned}
$$

Therefore,

$$
\begin{aligned}
\|T-Q\| & \leqq\left\|K_{\varepsilon}\right\|+\left\|T-K_{\varepsilon}-Q\right\| \\
& \leqq \varepsilon+\delta\left(\alpha\left\|T-K_{\varepsilon}\right\|+\beta+\frac{\left\|\left(T-K_{\varepsilon}\right)^{n}\right\|}{\alpha \beta^{n-1}}\right)
\end{aligned}
$$

and hence the theorem follows by choosing $\varepsilon$ small enough.

COROLLARY 3.2. Let $T$ be a biquasitriangular operator in $\mathscr{L}(\mathscr{C})$ such that $0 \in E(T)$. Then the distance $\operatorname{dist}(T, \mathscr{N}(\mathscr{H})) \leqq r(T)$.

Proof. From Theorem 3.1 we deduce that for an arbitrary $\varepsilon>0$ (taking $\alpha=\varepsilon, \beta=r(T)+\varepsilon$ and $\gamma=1+\varepsilon$ ) there exists a nilpotent operator $Q$ in $\mathscr{L}(\mathscr{H})$ such that

$$
\begin{aligned}
\| T & -Q \| \\
& \leqq(1+\varepsilon)\left\{\varepsilon\|T\|+r(T)+\varepsilon+\frac{1}{\varepsilon}(r(T)+\varepsilon)\left[\frac{\left\|T^{n}\right\|^{1 / n}}{r(T)+\varepsilon}\right]^{n}\right\} .
\end{aligned}
$$

Since $\lim _{n \rightarrow \infty}\left\|T^{n}\right\|^{1 / n}=r(T)$, it follows that for $n$ sufficiently large $\left[\| T^{n} \mid 1^{1 / n} /(r(T)+\varepsilon)\right]^{n}<\varepsilon^{2}$ and hence

$$
\|T-Q\|<(1+\varepsilon)[\varepsilon\|T\|+(r(T)+\varepsilon)(1+\varepsilon)] .
$$

Since $\varepsilon$ is arbitrary the proof of the corollary is complete.

The following corollary expresses the fact that if $T$ is a quasinilpotent operator on $\mathscr{H}$ and $\left\|T^{n}\right\|^{1 / n}$ doesn't decrease very fast, then there exists a sequence $\left\{Q_{n}\right\}$ in $\mathscr{N}(\mathscr{H})$ where each $Q_{n}$ has order $o_{n}, n=1,2, \cdots$ such that the rate of decrease of the sequence $\left\|T^{o_{n}}\right\|{ }^{1 / o_{n}}$ is the same as the rate of decrease of the sequence $\left\|T-Q_{n}\right\|$.

CoRollary 3.3. Let $T$ in $\mathscr{L}(\mathscr{H})$ be quasinilpotent and let 
$\delta>1$. Then there exists a sequence $\left\{Q_{n}\right\}$ in $\mathscr{N}(\mathscr{H})$ such that $Q_{n}^{4 n}=0$ and for some $c>0,\left\|T-Q_{n}\right\|<c\left(\|T\| \delta^{-n}+\left\|T^{n}\right\|^{1 / n}\right)$.

Proof. It is a direct consequence of Theorem 3.1 where $\alpha=\delta^{-n}$, $\beta=\delta\left\|T^{n}\right\| \|^{1 / n}$.

CoROLlaRY 3.4. Let $T$ in $\mathscr{L}(\mathscr{H})$ be quasinilpotent. Then there exists a sequence $\left\{Q_{n}\right\}$ in $\mathscr{N}(\mathscr{H})$ such that $Q_{n}^{4 n}=0$ and $\left\|T-Q_{n}\right\| \leqq$ $3(1+\|T\|)\left(n\left\|T^{n}\right\|\right)^{1 / n+1}$.

Proof. It is an immediate consequence of Theorem 3.1 taking $\alpha=\beta=\left(\left(n\left\|T^{n}\right\|\right) /(1+\|T\|)\right)^{1 / n+1}$ and $\gamma=3 / 2$.

In the next theorem we shall need the following terminology: If $X$ is a compact subset of the plane we denote by $\hat{X}$ the complement of the unbounded component of the complement of $X$. If $\varepsilon \geqq 0$ we denote by $X_{\varepsilon}$ the set $X_{\varepsilon}=\left\{\lambda \in C: \inf _{\mu \in X}|\lambda-\mu| \leqq \varepsilon\right\}$. We notice that if $\varepsilon \geqq \sup _{\lambda \in X}|\lambda|$, then $X_{\varepsilon}=(\hat{X})_{\varepsilon}=\left(X_{\varepsilon}\right)^{\wedge}$ and $0 \in X_{\varepsilon}$.

THEOREM 3.5. Let $T$ be in $\mathscr{L}(\mathscr{H})$. Then the distance $\operatorname{dist}(T, \mathscr{N}(\mathscr{H})) \leqq r(T)$.

Proof. Let $\varepsilon>0$ and let $\Sigma=\sigma(T) \backslash \widehat{E(T)_{\varepsilon}}$. If $\Sigma \neq \varnothing$, then $\Sigma=\left\{\lambda_{1}, \cdots, \lambda_{m}\right\}$ where each $\lambda_{j}$ is an isolated eigenvalue of $\sigma(T)$ such that $T-\lambda_{j}$ is Fredholm, $1 \leqq j \leqq m$. Then the spectral idempotent $E_{\Sigma}$ corresponding to $\Sigma$, associated with $T$, has finite rank. Let $\mathscr{F}=$ range $E_{\Sigma}, \mathscr{C}=\mathscr{F}^{\perp}$ and let $P$ be the projection from $\mathscr{H}$ onto $\mathscr{A l}$. It readily follows that $\sigma(T \mid \mathscr{F})=\sum$ and $\sigma(P T \mid \mathscr{M})=\sigma(T) \backslash \Sigma$. Furthermore, $E[(P T) \mid \mathscr{C}]=E(T)$ and $E_{l}[P T \mid \mathscr{M}]=E_{l}(T)$. Now we recall that a normal operator $M$ in $\mathscr{L}(\mathscr{H})$ is called diagonalizable if $\mathscr{H}$ has an orthonormal basis consisting of eigenvectors of $M$ and $M$ is said to have uniform infinite multiplicity if every eigenvalue of $M$ has infinite multiplicity. Notice that in this case $M$ is unitarily equivalent to $M \oplus M$ and that $\sigma(M)=E(M)$. From [3, Theorem 2.2] there exists a unitary transformation $U_{\varepsilon}: \mathscr{C} \rightarrow \mathscr{H} \oplus \mathscr{H}$ and a compact operator $K_{\varepsilon}$ on $\mathscr{C}$ whose norm is so small that $\left\|K_{\varepsilon}\right\|<\varepsilon$, $\sigma\left[P T \mid \mathscr{M}-K_{\varepsilon}\right] \subset \widehat{E(T)_{2 \varepsilon}}$ and such that we also have

$$
U_{\varepsilon}\left[P T \mid \mathscr{M}-K_{\varepsilon}\right] U_{\varepsilon}^{*}=\left[\begin{array}{ll}
M & R \\
0 & S
\end{array}\right],
$$

where $M$ is a diagonalizable normal operator of uniform infinite multiplicity in $\mathscr{L}(\mathscr{H})$ such that $\sigma(M)=E_{l}[P T \mid \mathscr{L}]$. Applying now the same reasoning to the operator $S$ we further deduce that there 
exist a unitary transformation $V_{\varepsilon}: \mathscr{L}_{s} \rightarrow \mathscr{H} \oplus \mathscr{H} \oplus \mathscr{H}$ and a compact operator $L_{\varepsilon}$ in $\mathscr{L}(\mathscr{C})$ with norm so small that $\left\|L_{s}\right\|<2 \varepsilon$, $\sigma\left[P T \mid \mathscr{L}-\mathscr{L}_{\varepsilon}\right] \subset \widehat{E(T)_{3 \varepsilon}}$ and such that

$$
T_{s}=V_{s}\left[P T \mid \mathscr{C}-P_{\varepsilon}\right] V_{\varepsilon}^{*}=\left(\begin{array}{ccc}
M & A & B \\
0 & C & D \\
0 & 0 & N
\end{array}\right),
$$

where $M$ is as before, $N$ is a diagonalizable normal operator of infinite uniform multiplicity in $\mathscr{C}(\mathscr{H}), A, B, C, D$ are in $\mathscr{P}(\mathscr{H})$ and $\sigma(N)=E_{r}(C)=E_{r}\left(\begin{array}{ll}C & D \\ 0 & N\end{array}\right) \subset E_{r}[P T \mid \mathscr{C l}]$. Now let $\delta=r(M), \eta=r(N)$ and notice that $\eta \leqq \delta$. From the remark preceding the present theorem we see that $\sigma(M)_{\delta}=\widehat{\sigma(M)_{j}}, \sigma(N)_{\eta}=\widehat{\sigma(N)_{\eta} \subset \sigma(M)_{\hat{o}}}$ and $0 \in \sigma(N)_{r}$. Employing a minor variation of [3, Proposition 1.11] it follows that there exist two normal operators $M^{\prime}$ and $N^{\prime}$ in $\mathscr{L}(\mathscr{H})$ such that $\sigma\left(M^{\prime}\right)=E\left(M^{\prime}\right)=\sigma(M)_{\dot{o}+3 \varepsilon}=\widehat{E_{l}(T)_{j+3 z}}=\widehat{E(T)_{j+3 \varepsilon}}, \quad \sigma\left(N^{\prime}\right)=$ $E\left(N^{\prime}\right)=\sigma(N)_{\eta}$ and $\left\|M-M^{\prime}\right\|=\delta+3 \varepsilon,\left\|N-N^{\prime}\right\|=\eta$. Let $T_{\varepsilon}^{\prime}$ be the $3 \times 3$ operator matrix defined by

$$
T_{\varepsilon}^{\prime}=\left(\begin{array}{lll}
M^{\prime} & A & B \\
0 & C & D \\
0 & 0 & N^{\prime}
\end{array}\right)
$$

We claim that $E_{l}\left(T_{\varepsilon}^{\prime}\right) \cap E_{r}\left(T_{\varepsilon}^{\prime}\right)=\sigma\left(T_{\varepsilon}^{\prime}\right)=\sigma\left(M^{\prime}\right)$. In order to prove this assertion we first observe that, since $M^{\prime}$ acts on an invariant subspace of $T_{\varepsilon}^{\prime}$, it is obvious that $\sigma\left(M^{\prime}\right)=E_{l}\left(M^{\prime}\right) \subset E_{l}\left(T_{\varepsilon}^{\prime}\right)$. Also, if $\lambda \notin E_{r}\left(T_{\varepsilon}^{\prime}\right)$, then $\lambda \notin E_{r}\left(\begin{array}{cc}C & D \\ 0 & N^{\prime}\end{array}\right)$ and hence $\lambda \notin E_{r}\left(N^{\prime}\right)=\sigma\left(N^{\prime}\right)$. Since $E_{l}(C) \subset \widehat{E_{r}(C)}$ and $E_{r}(C)=\sigma(N) \subset \sigma\left(N^{\prime}\right)=\widehat{\sigma\left(N^{\prime}\right)}$, it follows that $\lambda \notin E(C)\left(=E_{r}(C) \cup E_{l}(C)\right)$. Therefore $\lambda \notin E\left(\begin{array}{cc}C & D \\ 0 & N^{\prime}\end{array}\right)$, and since $\lambda \notin E_{r}\left(T_{\varepsilon}^{\prime}\right)$ we deduce that $\lambda \notin E_{r}\left(M^{\prime}\right)=\sigma\left(M^{\prime}\right)$. Now we shall prove the other inclusion. To this end we note that $\sigma\left(\begin{array}{ll}C & D \\ 0 & N\end{array}\right) \subset \widehat{\sigma\left(T_{\varepsilon}\right) \subset \widehat{E(T)_{3}}}$, and hence $\sigma\left(\begin{array}{ll}C & D \\ 0 & N\end{array}\right) \subset \widehat{E(T)_{\delta+3 \varepsilon}}=\sigma\left(M^{\prime}\right)$. In particular, it follows that $\sigma(C) \subset \sigma\left(M^{\prime}\right)$. Thus, if $\lambda \notin \sigma\left(M^{\prime}\right)$, then $\lambda \notin \sigma(C) \cup \sigma\left(N^{\prime}\right)$ and hence

$$
\lambda \notin \sigma\left(\begin{array}{lll}
M^{\prime} & A & B \\
0 & C & D \\
0 & 0 & N^{\prime}
\end{array}\right)=\sigma\left(T_{\varepsilon}^{\prime}\right) .
$$

The last observation establishes our claim. Let $\gamma=\operatorname{dist}\left(T_{s}^{\prime}, \mathscr{N}(\mathscr{C} \oplus\right.$ $\mathscr{H} \oplus \mathscr{H}))$. Our first conclusion is that $\operatorname{dist}(P T \mid \mathscr{M}, \mathscr{N}(\mathscr{M})) \leqq$ $\left\|L_{\varepsilon}\right\|+\operatorname{dist}\left(T_{\varepsilon}, \mathscr{N}(\mathscr{L} \oplus \mathscr{H} \oplus \mathscr{H})\right) \leqq 2 \varepsilon+\gamma+\max \left(\left\|M-M^{\prime}\right\|\right.$, $\left.\| N-N^{\prime}\right)$. Since $\left\|M^{\prime}-M\right\|=\delta+3 \varepsilon \leqq r(T)+3 \varepsilon$ and $\left\|N^{\prime}-N\right\|=$ 
$\eta \leqq \delta$, we deduce that dist $(P T \mid \mathscr{A}, \mathscr{N}(\mathscr{C l})) \leqq \gamma+r(T)+5 \varepsilon$. Using the fact that $\mathscr{F}$ is finite dimensional it is elementary to check that $\operatorname{dist}(T \mid \mathscr{F}, \mathscr{N}(\mathscr{F})) \leqq r(T \mid \mathscr{F}) \leqq r(T)$ and hence we conclude that

$$
\begin{aligned}
& \operatorname{dist}\left(T, \mathscr{N}^{-}(\mathscr{H})\right) \leqq \max [\operatorname{dist}(T \mid \mathscr{F}, \mathscr{N}(\mathscr{F})), \\
& \operatorname{dist}(P T \mid \mathscr{L}, \mathscr{N}(\mathscr{C}))] \leqq \gamma+r(T)+5 \varepsilon .
\end{aligned}
$$

Since $0 \in E\left(T_{\varepsilon}^{\prime}\right)$ and we have shown that $T_{\varepsilon}^{\prime}$ is a biquasitriangular operator we may conclude from Corollary 3.2 that $\operatorname{dist}(T, \mathscr{N}(\mathscr{Z})) \leqq$ $r\left(T_{\varepsilon}^{\prime}\right)+r(T)+5 \varepsilon \leqq 2 r(T)+8 \varepsilon$. However, we are interested in a sharper estimate. At this point we make use of the fact that $T_{\varepsilon}^{\prime}$ enjoys the further property that $\sigma\left(T_{\varepsilon}^{\prime}\right)$ is simple connected and coincides with $E\left(T_{\varepsilon}^{\prime}\right)$ and therefore from [4, Proposition 1.6], $\gamma=0$. Thus, $\operatorname{dist}(T, \mathscr{N}(\mathscr{C})) \leqq r(T)+5 \varepsilon$ and since $\varepsilon$ is arbitrary the proof of the theorem is complete.

4. Concluding remarks. In this section we pose some problems concerning the nilpotent approximation of quasinilpotent operators. We motivate these problems by presenting some pertinent observations.

Proposition 4.1. Let $T$ be in $\mathscr{L}(\mathscr{H})$ and suppose that there exists a sequence $\left\{Q_{n}\right\}$ in $\mathscr{N}(\mathscr{H})$ such that $Q_{n}^{n^{n}}=0, n=1,2, \cdots$ and $\lim \inf _{n \rightarrow \infty}\left\|T-Q_{n}\right\|^{1 / o_{n}}=0$. Then $T$ is quasinilpotent.

Proof. Since $T^{o_{n}}=T^{o_{n}}-Q_{n}^{o_{n}}=\sum_{j=1}^{o_{n}} T^{o_{n}^{-j}}\left(T-Q_{n}\right) Q_{n}^{j-1}$ then for $n$ sufficiently large there exists $c>0$ such that $\left\|T^{o_{n}}\right\|\left\|^{1 / o_{n}} \leqq\right\| T-Q_{n} \|^{1 / o_{n}}$. $\left[\sum_{j=1}^{o_{n}}\|T\|^{o_{n}-j}\left\|Q_{n}\right\|^{j-1}\right]^{1 / o_{n}} \leqq c\left\|T-Q_{n}\right\|^{1 / o_{n}}$. This completes the proof of the proposition.

Problem 1. Is the converse of Proposition 4.1 valid? (We expect a negative answer.)

REMARK 4.2. In a different circle of ideas we note that minor modifications of the arguments given in this paper show that if we replace $\mathscr{L}(\mathscr{H})$ by the Calkin algebra over $\mathscr{H}$ (i.e., the quotient algebra of $\mathscr{L}(\mathscr{H})$ by the ideal of compact operators on $\mathscr{H})$, then the conclusion of Theorem 3.5 still holds. Thus it is natural to ask:

Problem 2. If $\mathscr{A}$ is a $C^{*}$-algebra and $\mathscr{N}$ is the set of nilpotent elements of $\mathscr{A}$, when does it follow that the $\operatorname{distance} \operatorname{dist}(A, \mathscr{N}) \leqq$ $r(A)$, for every $A \in \mathscr{A}$ ?

If $\mathscr{A}$ is a finite type I von Neumann algebra, it is easy to see 
that the above question has an affirmative answer. On the other hand, it is worth noting that, by a well known fact in the theory of $C^{*}$-algebras [8], any noncommutative $C^{*}$-algebra contains nonzero nilpotent elements. However, if $\mathscr{B}$ is a semi-simple Banach algebra, then there exists no nilpotent element different from zero. In particular, if $\mathscr{B}$ is a semi-simple commutative Banach algebra such that the Gelfand transformation is not an isometry, then the corresponding version of the above question has a negative answer. This is the reason that in the preceding problem we imposed the condition that $\mathscr{A}$ be a $C^{*}$-algebra.

The following terminology was introduced in [10].

Definition. Let $T \in \mathscr{L}(\mathscr{H})$. We say that $T$ is pseudonilpotent if for every $\varepsilon>0$ there exists a finite orthogonal family of projections $P_{1}, \cdots, P_{n}$ in $\mathscr{L}(\mathscr{H})$ such that $\sum_{k=1}^{n} P_{k}=1$ and $\left\|\sum_{i \geqq j} P_{i} T P_{j}\right\|<\varepsilon$. The set of all pseudonilpotent operators in $\mathscr{L}(\mathscr{H})$ will be denoted by $\Psi(\mathscr{H})$.

In [10], it was observed that $\Psi(\mathscr{H})=\Psi(\mathscr{C})^{*}$ and it was shown that $\mathscr{N}(\mathscr{H}) \subset_{\neq} \Psi(\mathscr{H}) \subset_{\neq} \overline{\mathscr{N}(\mathscr{H})}$ and that there are pseudonilpotent operators which are not quasinilpotent.

LEMMA 4.3. Let $T \in \mathscr{L}(\mathscr{H})$ and suppose that there exists a sequence $\left\{Q_{n}\right\}$ in $\mathscr{N}(\mathscr{H})$ such that $Q_{n}^{o_{n}}=0, n=1,2, \cdots$ and $\lim \inf _{n \rightarrow \infty} \sqrt{o_{n}}\left\|T-Q_{n}\right\|=0$. Then $T$ is pseudonilpotent.

Proof. Given $\varepsilon>0$, let $n$ be sufficiently large so that $\sqrt{o_{n}}\left\|T-Q_{n}\right\|<\varepsilon$. For each $1 \leqq j \leqq o_{n}$ let $P_{j}$ be the projection onto kernel $Q_{n}^{j}$ kernel $Q_{n}^{j-1}$. Also let $x \in \mathscr{H}$. Then

$$
\begin{aligned}
\| \sum_{j=1}^{o_{n}} \sum_{i=j}^{o_{n}} P_{i} T P_{j} x_{\|} & =\left\|\sum_{j=1}^{o_{n}} \sum_{i=j}^{o_{n}} P_{i}\left(T-Q_{n}\right) P_{j} x\right\| \\
& \leqq \sum_{j=1}^{o_{n}}\left\|\left(\sum_{i=j}^{o_{n}} P_{i}\right)\left(T-Q_{n}\right) P_{j}\right\|\left\|P_{j} x\right\| \\
& \leqq\left\|T-Q_{n}\right\| \sum_{j=1}^{o_{n}}\left\|P_{j} x\right\| \leqq\left\|T-Q_{n}\right\| \sqrt{o_{n}}\|x\| .
\end{aligned}
$$

Therefore $\left\|\sum_{j=1}^{o_{n}} \sum_{i=j}^{o_{n}} P_{i} T P_{j}\right\| \leqq \sqrt{o_{n}}\left\|T-Q_{n}\right\|<\varepsilon$, as desired.

Proposition 4.4. Let $T$ be in $\mathscr{L}(\mathscr{H})$ such that

$$
\liminf _{n \rightarrow \infty} \sqrt{n}\left\|T^{n}\right\|^{1 / n}=0 \text {. }
$$

Then $T$ is pseudonilpotent and quasinilpotent. 
Proof. It is an immediate consequence of Corollary 3.3 and Lemma 4.3.

REMARK 4.5. Let $\left\{e_{n}\right\}$ be an orthonormal basis for $\mathscr{\mathscr { C }}$ and let $\left\{\alpha_{n}\right\}$ be a sequence of complex numbers. We recall that an operator $T$ in $\mathscr{L}(\mathscr{H})$ is called a unilateral weighted shift with weights $\alpha_{n}$, $n=1,2, \cdots$ if $T e_{n}=\alpha_{n} e_{n+1}$. Now we let $T$ in $\mathscr{L}(\mathscr{H})$ be a unilateral weighted shift with weights $\alpha_{o}=\alpha_{1}=\cdots=\alpha_{n_{1}}=1, \alpha_{n_{1}+1}=\cdots=$ $\alpha_{n_{2}}=1 / 2, \cdots, \quad \alpha_{n_{k-1}+1}=\alpha_{n_{k}}=1 / k, \cdots$, where $\left\{n_{k}\right\}$ is a strictly increasing sequence of natural numbers. Given an arbitrary slowly increasing sequence $\left\{c_{n}\right\}$ of real numbers $c_{n}>1$, tending to infinite, it is not difficult to define the sequence $\left\{n_{k}\right\}$ inductively so that $\lim _{k \rightarrow \infty} c_{n_{k}} / k=\infty$. It follows that for $k>1$, if $n_{k-1}<n \leqq n_{k}$, then $c_{n}\left\|T^{n}\right\|^{1 / n}>c_{n_{k-1}}\left\|T^{n_{k}}\right\|^{1 / n_{k}}>c_{n_{k-1}} k^{-1}$. Since $T$ is a quasinilpotent compact operator, from [10, Theorem 3.4], $T \in \Psi(\mathscr{H})$ but

$$
\lim _{n \rightarrow \infty} c_{n}\left\|T^{n}\right\|^{1 / n}=\infty \text {. }
$$

This shows that the sufficient condition of Proposition 4.4 (or any reasonable relaxation of it) is not necessary. Moreover, $T \otimes 1_{\mathscr{x}}$ provides a similar example with a noncompact quasinilpotent and pseudonilpotent operator.

Finally, we pose two questions concerning pseudonilpotent operators which were already asked in [10].

Problem 3. Is every quasinilpotent operator pseudonilpotent?

REMARK 4.6. From results of [3] and [4] it follows that every operator in $\mathscr{L}(\mathscr{H})$ has a nontrivial invariant subspace if and only if every operator in $\overline{\mathscr{N}(\mathscr{H})}$ has the same property.

Problem 4. Does every pseudonilpotent operator have a nontrivial invariant subspace?

\section{REFERENCES}

1. C. Apostol, On the norm-closure of nilpotents, Rev. Roum. Math. Pures et Appl., 19 (1974), 277-282.

2. C. Apostol and C. Foias, On the distance to the biquasitriangular operators, Annales Univ. Bucharest, 1974.

3. C. Apostol, C. Foias and D. Voiculescu, Some results on non-quasitriangular operators, IV. Rev. Roum. Math. Pures et Appl. 18 (1973), 487-514.

4. - On the norm closure of nilpotents, II, Rev. Roum. Math. Pures et Appl., 19 (1974), 549-577.

5. C. Apostol and D. Voiculescu, On a problem of Halmos, Rev. Roum. Math. Pures et Appl., 19 (1974), 283-284. 
6. P. R. Halmos, Ten problems in Hilbert space, Bull. Amer. Math. Soc., 76 (1970), 887-933.

7. D. A. Herrero, Toward a spectral characterization of the set of norm limits of nilpotent operators, Indiana Univ. Math. J., 24 (1974/75), 847-864.

8. I. Kaplansky, Rings of Operators, W. A. Benjamin, Inc., New York-Amsterdam, 1968.

9. N. Salinas, Reducing essential eigenvalues, Duke Math. J., 40 (1973), 561-580.

10. - Subnormal limits of nilpotent operators, Acta Sci. Math. (Szeged), 37 (1975), 117-124.

11. D. Voiculescu, Norm-limits of algebraic operators, Rev. Roum. Math. Pures et Appl., 19 (1974), 371-376.

Received June 12, 1975. During the preparation of this article the second author was partially supported by a grant of the University of Kansas General Research Fund.

Institute of Mathematics, Bucharest

AND

University of Kansas, Lawrence, Kansas 



\section{PACIFIC JOURNAL OF MATHEMATICS}

\section{EDITORS}

RICHARD ARENS (Managing Editor)

University of California

Los Angeles, California 90024

\author{
R. A. Beaumont \\ University of Washington \\ Seattle, Washington 98105
}

\section{J. DugundjI}

Department of Mathematics University of Southern California Los Angeles, California 90007

D. Gilbarg and J. Milgram

Stanford University

Stanford, California 94305

\section{ASSOCIATE EDITORS}
E. F. BECKENBACH
B. H. NeumanN
F. WOLF
K. YoshidA

\section{SUPPORTING INSTITUTIONS}

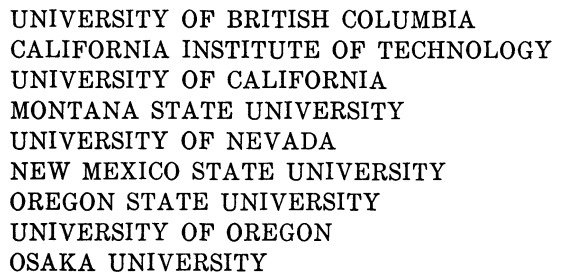

UNIVERSITY OF BRITISH COLUMBIA CALIFORNIA INSTITUTE OF TECHNOLOGY UNIVERSITY OF CALIFORNIA MONTANA STATE UNIVERSITY UNIVERSITY OF NEVADA NEW MEXICO STATE UNIVERSITY OSAKA UNIVERSITY OREGON STATE UNIVERSITY UNIVERSITY OF OREGON

\author{
UNIVERSITY OF SOUTHERN CALIFORNIA \\ STANFORD UNIVERSITY \\ UNIVERSITY OF TOKYO \\ UNIVERSITY OF UTAH \\ WASHINGTON STATE UNIVERSITY \\ UNIVERSITY OF WASHINGTON \\ AMERICAN MATHEMATICAL SOCIETY
}

The Supporting Institutions listed above contribute to the cost of publication of this Journal, but they are not owners or publishers and have no responsibility for its content or policies.

Mathematical papers intended for publication in the Pacific Journal of Mathematics should be in typed form or offset-reproduced, (not dittoed), double spaced with large margins. Please do not use built up fractions in the text of your manuscript. You may however, use them in the displayed equations. Underline Greek letters in red, German in green, and script in blue. The first paragraph or two must be capable of being used separately as a synopsis of the entire paper. Items of the bibliography should not be cited there unless absolutely necessary, in which case they must be identified by author and Journal, rather than by item number. Manuscripts, in triplicate, may be sent to any one of the editors. Please classify according to the scheme of Math. Reviews, Index to Vol. 39. All other communications should be addressed to the managing editor, or Elaine Barth, University of California, Los Angeles, California, 90024.

The Pacific Journal of Mathematics expects the author's institution to pay page charges, and reserves the right to delay publication for nonpayment of charges in case of financial emergency.

100 reprints are provided free for each article, only if page charges have been substantially paid. Additional copies may be obtained at cost in multiples of 50 .

The Pacific Journal of Mathematics is issued monthly as of January 1966. Regular subscription rate: $\$ 72.00$ a year $(6$ Vols., 12 issues). Special rate: $\$ 36.00$ a year to individual members of supporting institutions.

Subscriptions, orders for back numbers, and changes of address should be sent to Pacific Journal of Mathematics, 103 Highland Boulevard, Berkeley, California, 94708.

PUBLISHED BY PACIFIC JOURNAL OF MATHEMATICS, A NON-PROFIT CORPORATION

Printed at Kokusai Bunken Insatsusha (International Academic Printing Co., Ltd.), 8-8, 3-chome, Takadanobaba, Shinjuku-ku, Tokyo 160, Japan. 


\section{Pacific Journal of Mathematics}

\section{Vol. 61, No. 2 December, 1975}

Graham Donald Allen, Francis Joseph Narcowich and James Patrick Williams, An operator version of a theorem of Kolmogorov .......................

Joel Hilary Anderson and Ciprian Foias, Properties which normal operators share with normal derivations and related operators . . . . . . . . . . . . . . . . . . . . .

Constantin Gelu Apostol and Norberto Salinas, Nilpotent approximations and

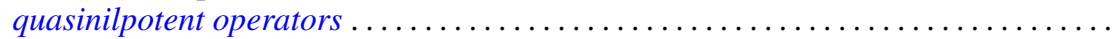

James M. Briggs, Jr., Finitely generated ideals in regular $F$-algebras . . . . . . . . . . .

Frank Benjamin Cannonito and Ronald Wallace Gatterdam, The word problem and power problem in 1-relator groups are primitive recursive ..................

Clifton Earle Corzatt, Permutation polynomials over the rational numbers ...........

L. S. Dube, An inversion of the $S_{2}$ transform for generalized functions . . . . . . . . . . William Richard Emerson, Averaging strongly subadditive set functions in unimodular

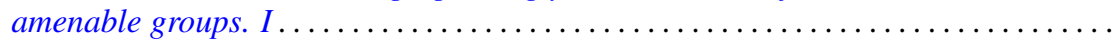

Barry J. Gardner, Semi-simple radical classes of algebras and attainability of

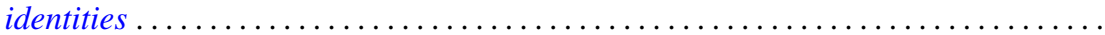

Irving Leonard Glicksberg, Removable discontinuities of A-holomorphic functions ....

Fred Halpern, Transfer theorems for topological structures . . . . . . . . . . . . . . .

H. B. Hamilton, T. E. Nordahl and Takayuki Tamura, Commutative cancellative

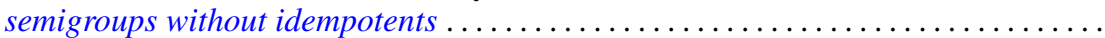

Melvin Hochster, An obstruction to lifting cyclic modules .....................

Alistair H. Lachlan, Theories with a finite number of models in an uncountable power

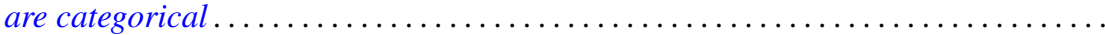

Kjeld Laursen, Continuity of linear maps from $C^{*}$-algebras . . . . . . . . . . . . .

Tsai Sheng Liu, Oscillation of even order differential equations with deviating arguments ....

Jorge Martinez, Doubling chains, singular elements and hyper- $Z$

Mehdi Radjabalipour and Heydar Radjavi, On the geometry of num Thomas I. Seidman, The solution of singular equations, I. Linear equations in Hilbert

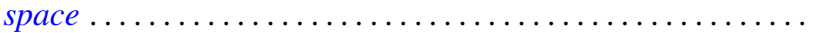

R. James Tomkins, Properties of martingale-like sequences ......

Alfons Van Daele, A Radon Nikodým theorem for weights on von Neumann

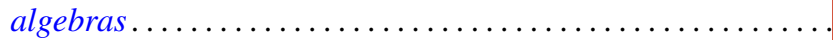

Kenneth S. Williams, On Euler's criterion for quintic nonresidues

Scott Andrew Wolpert, Noncompleteness of the Weil-Petersson metric for Teichmüller

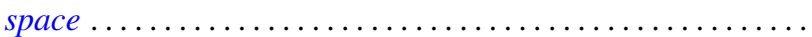

Volker Wrobel, Some generalizations of Schauder's theorem in locally convex

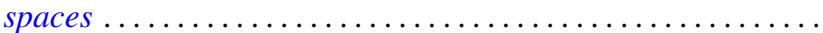

Kelly Denis McKennon, Corrections to: "Multipliers of type $(p, p)$ "; "Multipliers of type $(p, p)$ and multipliers of the group $L_{p}$-algebras"; "Multipliers and the

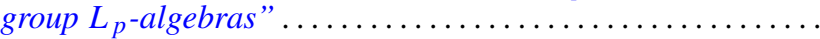

Andrew M. W. Glass, W. Charles (Wilbur) Holland Jr. and Stephen H. McCleary, Correction to: " $a *$-closures to completely distributive lattice-ordered

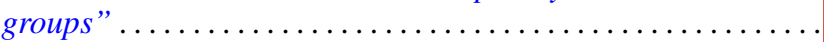

Zvi Arad and George Isaac Glauberman, Correction to: "A characteristic subgroup of

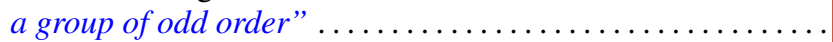

Roger W. Barnard and John Lawson Lewis, Correction to: "Subordination theorems

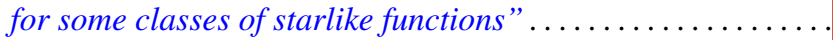

